\title{
Media e diritto: circolo virtuoso o vizioso?
}

\section{Media and law: vicious or virtuous circle?}

Maristella Amisano ${ }^{1}$

Resumo: Questo lavoro si pone l'obiettivo di indagare i rapporti tra media e diritto sotto un duplice profilo. In primo luogo cerca di comprendere se i media contribuiscono ad individuare -o addirittura si spingono a creare- gli interessi considerati meritevoli di tutela tanto da influenzare la politica legislativa. In secondo luogo vuole descrivere le storture del processo mediatico e la sua influenza sul processo rituale. Alla fine della duplice analisi si potrà concludere sul ruolo, positivo o negativo, che i media svolgono all'interno dell'ordinamento penale.

Palavras-chave: media, diritto penale, politica criminale, processo

1 Doutora (1995) pela Universidade de Turim (Itália), em Filosofia do Direito. PHD pela Adelphy University de Nova York com foco especifico nos crimes seriais. Advogada. Tem especializações em Direito penal minoril e Direito penal militar. Desde 2003 é professora na Faculdade de Direito da Università della Calabria. Membro do comitato científico do doutorado "Impresa, Stato, mercato" dell'Università della Calabria. Professora nos cursos de especilização da Ordem dos Advogados e referee do Ministério da Universidade e Pesquisa do Estado Italiano (MIUR). Colabora com várias universidades brasileiras. E-mail: maristami@libero.it 
Resume: This work aims to investigate the relationship between media and law in two ways. Firstly, it seeks to understand whether the media contribute to identifying - or even pushing to create - the interests considered worthy of protection so much as to influence legislative policy. Secondly, he wants to describe the distortions of the media process and its influence on the ritual process. At the end of the double analysis it will be possible to conclude on the positive or negative role that the media play within the criminal justice system.

Keywords: media, criminal law, criminal policy, trial

L'uomo è più piccolo della verità ed è più grande dell'errore. Papa Giovanni XXIII

\section{Il rapporto tra comunicazione mediatica e diritto penale.}

L'evoluzione della società risolve problemi così come ne crea altri in un percorso, non sempre costante, di cambiamenti culturali, sociali, politici e, necessariamente, giuridici. La diffusione dei mezzi di comunicazione, in una con la rapidità che caratterizza nella società moderna il flusso informativo hanno portato a domandarsi se e come i mezzi di comunicazione possano influenzare il diritto e, nello specifico, il diritto penale. Per la verità, l'influenza è un dato ormai assodato. Tutto sta nel vedere quale tipo di influenza i media esercitino sul diritto penale.

Due mi paiono gli ambiti ed i modi in cui il diritto penale subisce una influenza mediatica. Ve ne sono anche 
altri ${ }^{2}$, tuttavia credo che valga la pena concentrarsi prevalentemente su questi due aspetti: come i media influenzano l'attività legislativa e come i mezzi di comunicazione influenzano, attraverso quello che è chiamato comunemente 'processo mediatico', l'applicazione della legge penale nella aule di giustizia.

Ci si rende subito conto dell'importanza degli ambiti in cui la comunicazione influenza il diritto penale, così come si avverte immediatamente che il flusso è univoco: è il diritto penale ad essere influenzato dalla comunicazione mediati$\mathrm{ca}$, non viceversa. Per lo meno nella maggior parte dei casi. Certo, esistono delle regole, delle norme penali che mirano a tutelare la corretta amministrazione della giustizia nonchè il diritto a determinate forme di riservatezza degli indagati e che quindi delineano gli illeciti commissibili attraverso $\mathrm{i}$ media. Ma non si tratta di influenza del diritto penale sui media, bensì di mera sottoposizione di tutti alle regole giuridiche vigenti. Per 'influenza', infatti, mi riferisco al fatto che gli approdi raggiunti nell' ambito di un settore abbiano rilevanza nell'altro, orientandone le scelte. In questo senso, il rapporto è sostanzialmente univoco e dai media va al diritto penale.

Data la diffusione degli strumenti mediatici, che non si limitano a televisione e carta stampata, ma che comprendono l'universo costituito dalla rete, è inevitabile che questa grande parte dell'opinione sociale eserciti sul diritto penale una qualche influenza. Quel che dobbiamo vedere è in che termini questa influenza si eserciti e se vengano rispettati i

2 Si pensi alla c.d. teoria ipodermica, secondo cui i messaggi mediatici colpiscono gli individui in modo diretto ed immediato e così li condizionano sino all'emulazione dei gesti violenti. Cfr. Wolf, Gli effetti sociali dei media, Strumenti Bompiani, Milano, 1992 
principi che presiedono al nostro ordinamento. Se è vero, infatti, che l'evoluzione della società e dei mezzi che questa caratterizzano ha inevitabile incidenza sul diritto, è altrettanto vero che il nostro ordinamento giuridico si basa su principi che fondano il nostro stesso consorzio sociale e che, quindi, non possono essere derogati. Più precisamente: non possono essere derogati ma possono essere cambiati qualora non rispondano più al comune sentire che oggi caratterizza l'ordine democratico. Ma siamo ancora ben lontani da questo punto. Ed allora la compatibilità con i principi diventa essenziale.

Una premessa di metodo: mi occuperò prima del modo in cui i media influenzano la politica legislativa e poi di come il processo mediatico influisca sul processo giudiziario. Benchè il processo mediatico e le sue conseguenze sulla giustizia celebrata nei tribunali abbiano un impatto emotivo maggiore, l'influenza mediatica sulle scelte del legislatore ha un significato ancora più pregnante: la questione da risolvere non è come i media distorcano la realtà, ma piuttosto come essi la costruiscano ${ }^{3}$.

Solo al termine tenterò alcune conclusioni sul rapporto media-diritto penale.

\section{Politica criminale e influenza dei media}

In via di prima approssimazione, per politica criminale possiamo intendere quella visione generale e sistematica del diritto penale che orienta le scelte del creatore legislativo ${ }^{4}$. A seconda dei beni che il legislatore considera meritevoli di tu-

3 In tal senso: Luhmann, La realtà dei mass media, Franco Angeli, Milano, 2000.

4 Per quanto riguarda il concetto di politica criminale, rimando ad autorevole dottrina: Roxin, Politica criminale e sistema del diritto penale, Esi, Napoli, 2001; Moccia, Politica criminale e riforma del sistema penale, Jovene, Napoli, 1984. 
tela e a seconda del tipo di sanzione che ritenga di attribuire ai comportamenti lesivi dei beni stessi, il legislatore pianifica il suo intervento normativo. Non vorrei essere inutilmente polemica ma ritengo che da ormai molto tempo il legislatore non abbia una visione a lungo raggio -o anche solo a medio raggio- dell'intervento normativo in materia penale. Le leggi sono emanate sull'onda emotiva dell' una piuttosto che dell'altra emergenza. Il che ha portato a parlare autorevole dottrina di emergenza continua ${ }^{5}$. Il legislatore, passando da emergenza ad emergenza, crea norme contingenti, senza adeguata pianificazione prospettica e molto spesso senza adeguato e necessario coordinamento. Come è chiaro, se l'emergenza diventa continua, perde le caratteristiche che la contraddistinguono per diventare normalità. Quel che resta è una farneticante proliferazione normativa che non si basa su un progetto, un'idea di fondo, ma resta legata alle contingenze temporanee. $E^{\prime}$ quel che accade quando le risposte del legislatore alle necessità sociali sono non adattative ${ }^{6}$, cioè viscerali: la giustizia diventa più soggetta ad influenze esterne ed agli umori sociali. In questo modo il decisionismo diventa populista, preoccupandosi più della risposta a breve termine del consenso popolare che delle soluzioni più efficaci benché impopolari. Arrivando al paradosso che si perde la politica legislativa a fronte di una legislazione politica.

Questo, purtroppo, da diversi anni, lo scenario che sta dietro alle scelte del legislatore. Occorre domandarsi che ruolo hanno, in tutto questo, i media.

5 Moccia, La perenne emergenza. Tendenze autoritarie nel sistema penale, Esi, Napoli, 2000

6 Riprendo la distinzione tra risposte adattative e non adattative di Garland, La cultura del controllo. Crimine e ordine sociale nel mondo contemporaneo, Il Saggiatore, 2007, 30ss. 
L'importanza dei media in questo tipo di processo legislativo è diventato ogni giorno più significativa, poiché i mass media rivestono grande influenza nella percezione sociale del crimine. Essi si propongono come specchio della realtà e quindi rappresentano il crimine, tanto da essere, nella società contemporanea, la principale fonte di conoscenza sociale della realtà criminale. $E^{\prime}$ l'opinione pubblica che spesso crea quell'emergenza che spinge il legislatore all' intervento. D'altronde la nostra società costruisce senso ed attribuisce significato prevalentemente sulla base dei contenuti mediali che vengono offerti ${ }^{7}$. L'opinione pubblica si forma attraverso i media e da questi arriva fino al legislatore. Sono i c.d. effetti a lungo termine dei media sul comportamento umano, ove l'impatto non è sul singolo ma assurge a livello sociale. I media determinano la nostra percezione mentale della realtà che ci circonda ${ }^{8}$ e ci muovono all'azione, non solo singola bensì sociale. E questo ha inevitabile incidenza sul diritto.

Per spiegarmi meglio, prenderò a parametro alcuni esempi recenti che mi paiono particolarmente significativi.

Nel $2016^{9}$ è stata varata la legge sull'omicidio stradale. Di lesioni o financo omicidi compiuti in violazione delle norme del codice della strada ne sono sempre avvenuti. Certo, sempre di più a causa della diffusione via via più massiccia delle automobili e del loro utilizzo. Ma la guida è attività intrinsecamente pericolosa, quindi non è una sor-

7 Certo, questo non avviene in modo del tutto meccanico e passivo: anche chi riceve le informazioni possiede una capacità di rielaborazione che dà luogo ad un feedback. In proposito si veda Silverstone, Perchè studiare $i$ media?, Il Mulino, Bologna, 2002, p. 7ss.

8 Cfr. Wolf, Teorie delle comunicazioni di massa, Strumenti Bompiani, 2001 e, soprattutto, Cheli, La realtà mediata. L'influenza dei mass media tra persuasione e costruzione sociale della realtà, Franco Angeli, Milano, 1992.

9 Con L. 23/3/2016 n. 41 è stato introdotta la fattispecie di omicidio stradale di cui all'art. 589 bis c.p. 
presa che possa dare luogo ad episodi lesivi. A questo si è aggiunto, una decina di anni fa, altro elemento frutto di un cambiamento della società o forse semplicemente il risultato di una presa d'atto: l'uso di sostanze alcooliche o stupefacenti che ha portato ad incidenti causati dalla guida in stato di ebbrezza o alterazione da assunzione di stupefacenti. In realtà, niente di completamente nuovo, se non fosse per l'eco mediatico che alcuni incidenti stradali dovuti all'assunzione di sostanze alcoliche o stupefacenti hanno avuto. L'idea che persone ubriache al volante potessero causare morti ha colpito -a ragione- $\mathrm{l}^{\prime}$ opinione pubblica, che ha iniziato a porre attenzione sulle sanzioni irrogate ai rei. Insomma, alla notizia dell'incidente e delle circostanze in cui era avvenuto non seguiva più il silenzio, sino all'incidente successivo, bensì i media hanno iniziato a seguire l'iter processuale e a dare atto delle sentenze, delle relative condanne ed anche della sorte del reo: se gli veniva applicata una misura cautelare, se la pena irrogata lo avrebbe portato in carcere e così via. A seguito di un periodo di grande attenzione dei media nei confronti del fenomeno è emerso un quadro di ritenuta -non a torto- insufficienza della giustizia penale: la pena comminata per l'omicidio colposo non sembrava essere sufficiente a sanzionare adeguatamente quei comportamenti scellerati al volante che lasciavano dietro di sè morti e feriti. Il disagio dell'opinione pubblica è passato nelle aule di giustizia, ove $c^{\prime}$ è stato un tentativo di adeguare la sanzione alla gravità dell' accaduto attraverso un ripensamento dell' elemento soggettivo che sorregge il fatto. In alcuni casi, si è ritenuto che l'elemento soggettivo non fosse la colpa bensì il dolo eventuale $^{10}$. Non voglio ripercorrere qui i termini giuridici della

10 Fra le molte, ricordiamo la pronuncia Cassazione Penale, Sez. I, 16 settembre 2015 (ud. 26 marzo 2015) n. 37606. Nella specie, un uomo ubriaco e sotto effetto di stupefacenti, nel tentativo di sfuggire ad un controllo di polizia, 
differenza tra dolo eventuale e colpa cosciente ${ }^{11}$. La questione è da sempre discussa, se non nei lineamenti teorici, dove l'accettazione del rischio è elemento di facile discrimine, nella pratica applicazione, ove la necessità di provare l'elemento soggettivo al pari di qualunque altro elemento costitutivo del fatto di reato complica le cose. Un esempio chiarirà meglio ciò che intendo dire e mostrerà anche l'inadeguatezza del tentativo operato dalla giurisprudenza per commisurare la sanzione alla gravità di determinati omicidi commessi in violazione delle norme del codice stradale. Oltre ad evidenziare l'importanza del ruolo dei media.

La realtà può superare la più fervida immaginazione. Un soggetto a cui già era stata ritirata la patente per essere stato colto a guidare sotto l'effetto di stupefacenti e che, pertanto non era abilitato in quel momento alla conduzione di veicoli, messosi ciononostante alla guida della sua Mercedes dopo aver nuovamente assunto sostanze alcooliche e stupefacenti, stava percorrendo un tratto strada urbana in centro città (Roma), con grande traffico di veicoli e persone, quando ha altresì iniziato un alterco con la fidanzata, passeggero al suo fianco. L'alterazione provocata dagli stupefacenti e dall'alcool nonché dallo stato emotivo dovuto al litigio

aveva investito ed ucciso un pedone. La Corte ha ritenuto che, pur sapendo che proseguendo in quella direzione avrebbe investito il pedone, l'agente non ha cambiato direzione, accettando così il rischio dell'evento dannoso. Molte altre sono le pronunce, spesso commentate dalla dottrina. Si veda, in proposito: Trib. di Roma 26/11/2008, Foro it., 2009, II, 414ss., con nota di Fiandaca, Sfrecciare col "rosso" e provocare un incidente mortale: omicidio con dolo eventuale?; Corte Appello Milano 16/7/2009, in Giurisprudenza di merito, 2010, 3, 757ss, con nota di Agnino, Colpa cosciente e dolo eventuale in tema di sinistri stradali; Gip Milano 21/4/2004, in Corriere di merito 2005, 70ss., con nota di Viganò, Fuga spericolata in autostrada e incidente con esito letale: un'ipotesi di dolo eventuale?

11 Per tutte, si veda la pronuncia a Sezioni Unite del 18/9/2014, n. 38343, emanata nel famoso caso ThyssenKrupp relativa alla distinzione tra dolo eventuale e colpa cosciente. 
hanno condotto il soggetto a percorrere a velocità elevata due incroci semaforici passandoli, senza neppure rallentare, con il segnale rosso. Al terzo incrocio, superato sempre con il segnale rosso e ad alta velocità, il veicolo è entrato in collisione con uno scooter, su cui viaggiavano due fidanzati di sedici anni. Lo scooter era regolarmente passato con il segnale verde ed è stato "spazzato" via dall' auto che arrivava ad alta velocità e che invece avrebbe dovuto essere ferma al rosso, come il guidatore dello scooter legittimamente si aspettava. I due ragazzi a bordo del ciclomotore sono morti, mentre l'agente è ancora riuscito a scappare ed a nascondere la vettura in una rimessa. Questo fatto, riportato da tutti i giornali a causa della gravità dell'episodio che ha cagionato due giovanissime vittime e che è stato il frutto di una serie innumerevole di atti sconsiderati, è diventato notorio. Come si usa dire: un caso mediatico. Per questo ha suscitato una forte eco l'esito del processo. Durante il giudizio, il pubblico ministero ha prospettato che il fatto del reo fosse sorretto non già da colpa cosciente bensì da dolo eventuale. La tesi dell'accusa si basava su dati di fatto. L'essersi messo alla guida nonostante il divieto, aver guidato sotto l'effetto di sostanze alcoliche e stupefacenti, avere guidato a velocità eccessiva senza fermarsi con i semafori rossi: erano tutti indicatori del fatto che l'agente non ha creduto di poter evitare il fatto con la propria abilità (atteggiamento psicologico tipico della colpa cosciente) ma che ha accettato il rischio del verificarsi dell'evento. Non colpa cosciente, quindi, bensì dolo eventuale. Secondo l'ipotesi dell' accusa, non si sarebbe dovuta applicare, quindi, la pena per l'omicidio colposo, ma quella per omicidio doloso. Pena che avrebbe consentito anche una sanzione soddisfacente per il rispetto dovuto alle vittime. La tesi accusatoria è stata accolta in primo grado, con la condanna a dieci anni di reclusione, mentre in appello 
è stato escluso il dolo eventuale e la pena è stata ridotta a cinque anni. La Corte di Cassazione, con riferimento a questo caso specifico, ha avallato la pronuncia di secondo grado, statuendo un principio che è stato oggetto di molte critiche anche da parte della dottrina penalistica. Secondo i giudici del Supremo Collegio, il comportamento del reo sarebbe stato tale da accettare il rischio di un incidente, ma non il rischio di un incidente mortale di quelle proporzioni. Non è il luogo, questo, per commentare una pronuncia dai contorni francamente discutibili. Ciò che conta è che, essendo stata rigettata la tesi del dolo eventuale, il reo è stato condannato per omicidio colposo alla pena di cinque anni. Pena ritenuta insufficiente dalle vittime e dall'opinione pubblica, che - come ho già sottolineato- aveva seguito la vicenda sin dall'inizio. Alternandosi pronunce che riconoscevano il dolo eventuale ed altre che applicavano la colpa cosciente, tutto era lasciato alla sensibilità dei giudici e questo ha generato grande disparità di trattamento. Disparità che ha portato le associazioni dei parenti delle vittime della strada ad iniziare una campagna di sensibilizzazione sul tema, finalizzata ad una presa di posizione legislativa verso pene più dure nei casi di omicidi stradali. La campagna di sensibilizzazione si è svolta prevalentemente col mezzo - mi sia permessa la tautologia-mediatico: per mesi, quotidianamente, su giornali e riviste era data notizia di morti sulle strade cagionate da comportamenti di persone ubriache, drogate, che non avevano la patente, che procedevano a velocità sproporzionata, che stavano sfuggendo a controlli di polizia e così via. Ecco che i media hanno contribuito a creare o, a tutto concedere, hanno dato risonanza ad una esigenza di riforma del sistema penale in materia. Il legislatore ha recepito questa necessità e vi ha provveduto con la legge del 2016 cui ho già accennato. 
Non è questa la sede per sottolineare le carenze di questa normativa ${ }^{12}$, che dipendono solo dalle scelte e dalla tecnica usata dal legislatore. Ciò che qui conta è che un interesse meritevole di intervento legislativo-penale è stato messo in evidenza dall'opera dei media.

Come possiamo valutare questo dato? $\mathrm{E}^{\prime}$ positivo o negativo che siano stati i media ad influenzare la riforma normativa?

Sgomberiamo il campo da un dato che potrebbe essere fuorviante. Il fatto che la riforma non sia stata la migliore possibile non è colpa dei media ma dipende dal solo legislatore. Ai media resta il ruolo di aver sollevato una situazione problematica fortemente presente tra i consociati. Ed allora la risposta passa attraverso un'altra questione: in che modo devono selezionarsi quegli interessi che orientano le riforme del sistema penale? Poichè sono interessi che debbono essere individuati all'interno del consorzio sociale e che devono venire incontro alle esigenze che in quel determinato momento storico la società manifesta, a mio sommesso parere nulla osta a che gli interessi siano quelli che si manifestano attraverso i mezzi di comunicazione. Questi ultimi, infatti, non fanno che svolgere il loro ruolo di mezzi, appunto. Nulla di strano, nè di illegittimo. Anzi, un modo al passo con i tempi di individuazione dei nodi dell'ordinamento che meritano di essere sciolti.

Ritorniamo al punto di partenza: quel che desta perplessità è l'azione del legislatore, priva di coordinamento e di programmazione e basata -ormai sempre- sulle continue emergenze.

12 Sono state riprese -anche dai media- le forti critiche alla L. 41del 2016 espresse dall'unione delle Camere Penali. Per le prime riflessioni, a ridosso dell'introduzione della nuova fattispecie delittuosa, si veda Squillaci, Ombre $e$ (poche) luci nella introduzione dei reati di omicidio e lesioni personali stradali, in www.dirittopenalecontemporaneo 18/4/2016. 
E gli esempi non si limitano all'omicidio stradale. Solo un cenno ad altra questione recente. Appena sanzionato dalla Corte europea il nostro Paese per i fatti avvenuti nella caserma Diaz ${ }^{13}$, ecco che appare un disegno di legge che abbozza una riforma modellata quasi pedissequamente su quanto è accaduto nella caserma Diaz e su tutto ciò che ne è seguito. Disegno legge che, dopo un lungo e tortuoso iter normativo, ha dato luogo alla legge 14/7/2017 n. 110 che ha inserito nel nostro ordinamento penale l'art. 613 bis: "Tortura". Norma molto criticata ${ }^{14}$, anche perché -appunto- non è stata sorretta da una adeguata riflessione politico-criminale ma ha semplicemente costituito lo specchio dell'occasione che l'ha originata.

In questo caso, accanto all'opera di sollecitazione dei media si sono unite considerazioni giuridiche, prevalentemente di natura internazionale. Ma ciò non toglie che $\mathrm{i}$ fatti della caserma Diaz siano stati oggetto dell'attenzione di stampa e televisione per molto tempo e che, quindi, la funzione mediatica abbia avuto un importante rilievo.

Possiamo dare una colpa di questo atteggiamento a chi fa opera di cronaca o che comunque si esprime su fatti di cronaca? Direi proprio di no. Si tratta di semplice processo di selezione di quegli interessi diffusi nel consorzio sociale

13 Con la sentenza Bartesaghi Gallo ed altri contro Italia, rg. 12131/13 e 43390/13, del 22 giugno 2017, la Corte europea dei diritti dell'uomo ha reiterato la condanna dell'Italia in relazione alle violenze della scuola Diaz occorse a margine del G8 di Genova nel luglio 2001. In particolare, la sentenza ha riconosciuto la violazione dell'articolo 3 della Convenzione sul piano sostanziale e procedurale ed ha condannato lo Stato italiano al risarcimento dei danni morali in favore di ciascun ricorrente.

14 Si veda, fra tutti, Manconi, Perchè la legge sulla tortura è un'occasione mancata, in L'espresso, 6/7/2017; Marchi, Il delitto di tortura: prime riflessioni a margine del nuovo art. 613 bis c.p., in www.dirittopenale contemporaneo, 31/7/2017, Lobba, Punire la tortura in Italia. Spunti ricostruttivi a cavallo tra diritti umani e diritto penale internazionale, in www. diritto penale contemporaneo, 31/10/2017. 
che vengono ritenuti meritevoli della tutela più forte: quella che si esercita col diritto penale. Attraverso un processo di circolarità gli interessi più significativi individuati all'interno della nostra società arrivano al legislatore che li tiene in considerazione nel suo processo legislativo. $E^{\prime}$ lo stesso meccanismo che da sempre ha orientato il diritto penale, solo che oggi si svolge con mezzi più moderni di comunicazione e quindi di individuazione. Non si può sottacere che i media svolgono una funzione selettiva che necessariamente influenza i fattori legislativi. Come è stato autorevolmente ${ }^{15}$ detto, di fronte alle notizie criminali ed al conseguente allarme sociale imperante, si generano richieste di criminalizzazione e di pene più severe da parte dell'opinione pubblica. Si vuole ridefinire ciò che è criminale e si vuole dare una nuova riformulazione della pena ${ }^{16}$. Di certo il sistema mediatico funge da suscitatore del consenso sociale in ambito di politica criminale e di collettore dei bisogni di pena. Il filtro che i media operano sulla realtà, attraverso la selezione di determinati aspetti del crimine su cui vengono espressi giudizi di valore, concorre a formare l'idea dei comportamenti che devono costituire reato e di come lo Stato si adoperi per combattere la delinquenza. Inevitabile che questo crei pressioni sullo Stato, perché ingenera nei consociati fiducia o sfiducia sulle capacità della giustizia penale di fronteggiare il crimine, dando ai cittadini quel tanto cercato senso di sicurezza ${ }^{17}$. E senza dubbio le capacità selettive, per non dire distorsive, dei media sono in grado di produrre o accentuare la discrasia tra la criminalità ufficiale e quella percepita. Il che significa che i media non si

15 Paliero, La maschera e il volto, in Riv. it. dir e proc pen., 2006, n. 2, p. 467ss.

16 Paliero, cit., p. 524.

17 Mi permetto di rinviare, per quanto concerne il sentimento di sicurezza percepito dai cittadini e la criminalità, a: Amisano, L'uso del linguaggio nella pericolosità. Concetto e applicazioni, Le Monnier, 2017, 231ss. 
limitano a raccogliere le esigenze presenti nella società ma le creano. Il problema principale, però, a mio parere, resta che il legislatore risponde alle pressioni con scelte simboliche, prive di visione a lungo raggio.

Quel che è certo è che vi è una forte incidenza dei media nella selezione di quegli interessi che risultano meritevoli della tutela penale.

Ma questo, come abbiamo visto nell'incipit, non è che uno degli aspetti che vede legati i media ed il diritto penale.

\section{Il cosiddetto processo mediatico: profili des- crittivi e critici.}

Vorrei partire da un dato piuttosto lontano: non è possibile rendersi compiutamente conto del significato di qualunque prescrizione giuridica, sostanziale o processuale, se non se ne ripercorrono i fattori genetici. Questi spesso sono lo specchio degli interessi che in quella determinata norma si sono espressi. Inizio questa seconda parte di questa indagine, quindi, riprendendo dallo stesso punto in cui ho concluso il paragrafo precedente. Le norme nascono a difesa di un interesse considerato bisognoso di tutela. Per queste ragioni la storia del processo penale è tanto importante: perché ci mostra le radici delle norme dalle quali ricaviamo che le regole del processo penale oggi vigenti sono il risultato di un percorso che ha attribuito all' individuo sempre più garanzie, ricercando la certezza del giudizio. Si badi che l'evoluzione in senso moderno del processo non va verso la certezza ma proprio verso le garanzie. Infatti, anche l'ordalia era uno strumento che assicurava la certezza del giudizio ${ }^{18}$ : nell' ordalia la certezza veniva da un'istanza superiore, che è la volontà di

18 Sorrentino, Storia del processo penale. Dall'ordalia all'inquisizione, Rubbettino, 1999 
Dio, ma era comunque assicurata. Quel che mancava erano le garanzie dei diritti dell'imputato, soprattutto con riguardo alla sua difesa. Il punto centrale che caratterizza il processo moderno è che la pretesa certezza del giudizio si unisce al rispetto delle garanzie che vanno riconosciute all'imputato. Ecco quindi che il processo di oggi è scandito da regole perché queste sono l'approdo raggiunto in tema di garanzie e di diritti dell'imputato nella logica della ricerca della verità. E sul tipo di verità che il processo ricerca tornerò in chiusura di queste poche righe.

Occorre tenere a mente la ragione per cui esistono le regole del processo giudiziario perché è noto a tutti che talvolta, in un binario parallelo a quello del processo penale, si instaura altro e diverso processo portato avanti dall'opinione pubblica attraverso i media. Ed è altrettanto noto come da più parti ${ }^{19}$ si siano sollevate grosse perplessità sulla legittimità di questo processo mediatico. Innanzitutto in quanto privo della necessaria osservanza delle regole tecniche: il che inevitabilmente viola le garanzie dell'imputato. Ma non solo. Abbiamo visto come l'opera dei media crei delle distorsioni, che possono essere quantitative, qualitative o contenutistiche. Dal punto di vista quantitativo, sono senza dubbio trattati dai media più i crimini violenti degli altri, benché siano percentualmente meno. E questo contribuisce a facilitare una falsa lettura della realtà da parte dei fruitori dei media. Le distorsioni qualitative, poi, portano ad una presentazione emotiva ed emotigena dei fenomeni rappresentati. Infine, le distorsioni contenutistiche sono quelle che più hanno a che fare col processo mediatico, perchè è attraverso questo tipo di distorsione che si fa dell'autore del reato un mostro. Considerazioni, tutte, che hanno fatto dire ${ }^{20}$ che

19 Cfr. Bertolino, La televisione del crimine, in Vita \& Pensiero, 2005

20 Garland, La cultura del controllo, cit., p. 268 
"la conoscenza e l'opinione pubblica intorno alla giustizia penale si fondano oggi su rappresentazioni collettive più che su un'informazione accurata; su un' esperienza della criminalità culturalmente connotata più che sul fenomeno in sè".

$\mathrm{E}^{\prime}$ del tutto evidente che il processo mediatico presenta aspetti critici di grande rilevanza, che mi limito a tratteggiare.

In primo luogo, nel processo mediatico chiunque può assumere un ruolo: tutti possono formulare giudizi e, quel che è peggio, possono formularli in piena libertà, arrivando a trascurare qualsivoglia diritto dell'imputato, primo fra tutti la presunzione di non colpevolezza. Il che porta ad una conseguenza di non poco conto, ovvero alla compromissione del contraddittorio, determinata dal fatto che molto spesso il lettore o lo spettatore tendono ad assumere passivamente le informazioni e sono portati così a ritenerle vere. Non ci sono elementi che insinuano il dubbio, non sono prospettate tesi alternative -almeno nella maggioranza dei casi.

Ma c'è un altro aspetto importante, evidenziato dalla lucida consapevolezza di Hannah Arendt, che aveva seguito con particolare interesse due grandi ed emblematici processi: quello di Norimberga ove era coinvolto anche Heidegger, accusato di aver favorito il regime nazista, che era il suo primo Maestro ma soprattutto il suo primo amore, e quello ad Eichmann. La grande lucidità che la Arendt è riuscita a mantenere assistendo a questi processi ha stimolato una importante riflessione filosofica ed ha portato alla produzione di alcune pagine tra le più belle della filosofia moderna. Ebbene, riprendendo l'idea romana della giustizia come Dea bendata, Hannah Arend $\mathrm{t}^{21}$ sostiene che giudicare impone di non vedere: solo chiudendo gli occhi si diventa spettatori imparziali. Operazione impossibile in un universo saturo di

21 Hannah Arendt, La banalità del male. Eichmann a Gerusalemme, Feltrinelli, 2013 
immagini com'è ormai il nostro. Insomma: giudicare con serenità impone di non vedere, è incompatibile con quel gioco di sguardi che mina imparzialità e terzietà. Invece il nostro sistema, grazie ai media, è pieno di immagini che fanno in modo che il processo esca dalle aule di giustizia e vi rientri influenzando i giudici dopo che il processo mediatico -con il suo linguaggio, con i suoi mezzi e con i suoi tempi ben più rapidi di quelli del dibattimento- è pervenuto ad una conclusione di colpevolezza o innocenza.

Ed è inevitabile che qualsiasi forma di conoscenza 'esterna' al processo provochi distorsioni selettive, rincorra il cosa pensa la gente e non realizzi un accertamento ponderato dei fatti.

E non basta ancora: ogni aspetto che concerne il diritto, in special modo il diritto penale, è materia su cui tutti ritengono di poter dire la loro. Siccome la criminalità è un fenomeno che tocca la società a tutti i livelli, diventa un argomento di senso comune, dove chiunque si sente autorizzato a dire la sua. Al contrario, il diritto è materia tecnica, talvolta di non facile comprensione, e spesso le ragioni tecniche contrastano con quelle di senso comune. Per questo nel processo mediatico accade di frequente che si seguano verità emotive, diverse da quella processuale, e si formi un convincimento collettivo destinato a radicarsi al punto che, se la sentenza formale non soddisfa le aspettative, si insinua addirittura il dubbio che la sentenza emanata nel processo giudiziario sia ingiusta. Il che genera insicurezza nei cittadini perché il delinquente non è più persona ma minaccia ${ }^{22}$. $E^{\prime}$ il fare del reo un mostro a cui ho accennato parlando delle distorsioni contenutistiche operate dai media.

22 Cfr. Amisano, L'uso del linguaggio nella pericolosità, cit., p. 231ss. e nella parte in cui descrive i pericoli del diritto penale del nemico, p.215ss. 
Facile, quindi, scagliarsi contro il processo mediatico in nome del rispetto delle garanzie che presiedono al processo penale. Facile parlare, usando l'espressione di Lariviere, di circo mediatico-giudiziario ${ }^{23}$.

$\mathrm{Ma}$, in realtà, le cose sono ben più complesse.

Innanzitutto perchè l'informazione è un diritto ed i giornalisti esercitano il loro diritto di cronaca ed anche di critica. I giornalisti svolgono una funzione pubblica che è quella della diffusione della notizia. Certo, bisognerebbe fare dei distinguo, che complicano le cose ulteriormente. Un conto è l'informazione che segue la notizia, altro sono quelle trasmissioni che imbastiscono processi paralleli fuori delle aule di giustizia, riproducendo col linguaggio mediatico i riti ed i simboli del processo. In questo secondo caso c'è un'indubbia lesione dei diritti dell' indagato o imputato. Tuttavia occorre riconoscere che il crimine incuriosisce da sempre. In molti processi della storia recente l'Italia si è divisa tra innocentisti e colpevolisti ed a questo ruolo non si sottraevano personaggi del calibro di Moravia o Buzzati. E dobbiamo riconoscere che queste trasmissioni hanno grande successo di pubblico, quindi di certo non si possono incolpare i giornalisti se danno al loro pubblico quello che richiede.

Fin qui sembrerebbe essere una questione di bilanciamento tra diritti. Ad esempio, la presunzione di innocenza ed il diritto di cronaca. Niente di nuovo: la Costituzione attribuisce un insieme di diritti che inevitabilmente entrano in conflitto e vanno quindi bilanciati. E sempre di bilanciamento di interessi si tratta quando si osserva che la funzione di rendere giustizia è tratto caratterizzante della sovranità dello $S$ tato $^{24}$ e, insieme al territorio ed alla popolazione, è un

23 Ora l'espressione 'circo' è addirittura stata sostituita da quella di 'inferno mediatico'.

24 Pittaro, Mass media e diritto penale. Profili di diritto sostanziale, in Rassegna 
elemento costitutivo dello Stato. Ne discende che se amministrare la giustizia rientra nel concetto di sovranità dello Stato, i primi articoli della Carta fondamentale pongono il principio che "la sovranità appartiene al popolo, che la esercita nelle forme e nei limiti della Costituzione" e che "la giustizia è amministrata in nome del popolo". La nostra Costituzione pone al centro l'uomo cui attribuisce una serie di diritti, non lo Stato. Il che significa che sia la giustizia penale, sia la libera manifestazione del pensiero espressa dai giornalisti sono in funzione del bene-persona, uti singulus e uti socius. Ecco che, in ipotesi di conflitto, si impone la ricerca di un punto di equilibrio.

Ma non si tratta solo di bilanciamento di interessi: un altro dato deve necessariamente essere preso in considerazione. Il processo mediatico non è opera soltanto dei giornalisti ma ha origine a seguito di molti comportamenti, tutti lesivi dei diritti dell'indagato, che vengono da attori diversi. Ad esempio, le forze di polizia spesso rilasciano conferenze stampa presentando ipotesi investigative come se fossero sentenze definitive. I giornalisti spessissimo basano i loro articoli sulle veline sfuggite ai magistrati, malgrado i divieti di pubblicazione degli atti sino alla conclusione delle indagini preliminari.

E gli avvocati difensori non sono alieni da questo meccanismo perché adorano partecipare alle trasmissioni che danno vita al processo mediatico, nascondendosi dietro il dito del dare voce al proprio assistito ma in realtà rispondendo a esigenze dettate dal proprio ego. Ed allora non possiamo dare la colpa delle storture solo ai giornalisti quando i protagonisti del tanto vituperato processo mediatico sono spesso gli stessi protagonisti del processo che si svolge nella sede appropriata. C'è poi un dato niente affatto trascurabile:

dell'arma dei carabinieri, 2009 
il processo mediatico assume funzioni socialmente utili. Per esempio, aiuta a valutare l'impatto sociale del lavoro del giudice: elemento importantissimo visto che la giustizia è esercitata in nome del popolo.

\section{Le norme che regolano la materia.}

Le osservazioni che precedono non si riferiscono ad un far west, un terreno privo di regole in cui tutti i protagonisti si muovono senza legge. Le regole ci sono e riguardano il comportamento di tutti coloro che agiscono nel processo rituale, che da questo escono per approdare al processo mediatico.

Esistono per i giornalisti (ma non solo) norme che sanzionano penalmente la pubblicazione arbitraria di atti di un procedimento penale. L'art. 684 c.p. punisce: “Chiunque pubblica, in tutto o in parte, anche per riassunto o a guisa $\mathrm{d}^{\prime}$ informazione, atti o documenti di un procedimento penale, di cui sia vietata per legge la pubblicazione (...)". Mentre l'art. 685 sanziona l'indebita pubblicazione di notizie concernenti le decisioni penali: "Chiunque pubblica i nomi dei giudici, con l'indicazione dei voti individuali che ad essi si attribuiscono nelle deliberazioni prese in un procedimento penale è punito (...)". Si tratta di contravvenzioni, quindi di reati considerati bagatellari. L'interpretazione dell'art. 684 non è lineare perchè segue la struttura delle norme processuali che vietano la pubblicazione degli atti. Per questo la dottrina ha parlato di norma penale in bianco. Per dare un contenuto alla fattispecie di cui all'art. 684 c.p. occorre rifarsi all'art. 114 c.p.p., che pone due tipi di divieti di pubblicazione degli atti: un divieto assoluto ed uno che investe l'atto ma non il suo contenuto e che quindi è relativo. Ma occorre soprattutto rifarsi agli atti coperti da segreto, in un continuo richiamo alla materia processuale che rende difficile comprendere il precetto ${ }^{25}$.

25 La Corte costituzionale, con sentenza 3/12/1987 n. 457 ha stabilito che l'art. 
Nel 2016 le Sezioni Unite ${ }^{26}$ si sono pronunciate su questo tema, affermando che esiste un doppio filtro alla pubblicazione degli atti: esiste un divieto assoluto di pubblicazione fino a quando l'imputato non ne possa avere conoscenza e, comunque, non oltre la chiusura delle indagini preliminari; $c^{\prime}$ è poi un divieto relativo limitato ai soli elementi testuali, fino al termine dell' udienza preliminare e, se si prosegue col dibattimento, fino alla prinuncia di appello. Questo secondo divieto riguarda, appunto, il testo ma non il suo contenuto. I due divieti risentono della diversa funzione che l'art. 684 c.p. assume nelle differenti fasi processuali: nella fase delle indagini preliminari la ratio è quella di non compromettere l'acquisizione delle prove; nella seconda fase è quella di salvaguardare serenità e terzietà del giudicante.

684 c.p. sancisce un reato plurioffensivo. Lede, infatti, sia la riservatezza di soggetti deboli quali minori o arrestati; sia il rispetto del c.d. doppio fascicolo, che impedisce al giudice di vedere il fascicolo del Pubblico Ministero affinchè possa formarsi nel contraddittorio il proprio convincimento; sia la terzietà del giudice e la presunzione di innocenza.

26 In realtà la pronuncia a SS.UU. 3727 del 2016 ha sancito altri tre fondamentali principi, che, per la verità, a me non paiono tutti condivisibili. Il primo principio, contrastante con la sentenza della Corte Costituzionale n. 457 del 1987 (citata alla nota che precede) è quello per cui la fattispecie dell'art. 684 c.p. è un reato monoffensivo (e non plurioffesivo). Ratio della norma sarebbe, prima della conclusione delle indagini preliminari, quella di non compromettere il buon andamento delle stesse e, dopo tale momento, quello di salvaguardare i principi propri del processo accusatorio. Secondo principio è che nessuna autonoma pretesa risarcitoria può essere avanzata dalla parte coinvolta nel processo "perciò solo che sia stata violata la norma incriminatrice in discorso" (ovvero per la semplice violazione dell"art. 684 c.p.). Occorre provare la concreta lesione alla reputazione e riservatezza. Principio, questo, che invece mi pare ineccepibile se non si vuole pensare al reato come ad un illecito di mera disobbedienza. Ultimo principio affermato è che la portata della violazione, sotto il profilo della limitatezza e della marginalità della riproduzione testuale di un atto processuale, va apprezzata dal giudice di merito, di nuovo in applicazione del principio della necessaria offensività della condotta ascritta all'agente. 
Aldilà delle questioni interpretative, ciò che più colpisce è la natura contravvenzionale del reato, che non pare sufficientemente dissuasiva per i giornalisti che, pur di pubblicare atti coperti da segreto, ben potrebbero accettare il rischio di una condanna, magari oblazionabile. Oltretutto, quando soggetto attivo è il giornalista, si potrebbe appellare al diritto di cronaca, anche se fino ad ora l'orientamento di dottrina e giurisprudenza è stato quello di negare la scriminante, privilegiando il buon andamento della giustizia. Corretta, in questo modo, risulta l'interpretazione della fattispecie: l'esistenza del diritto di cronaca non significa che qualunque condotta sia lecita. In questo caso, il legislatore ha già effettuato il bilanciamento degli interessi costituzionalmente rilevanti e che si trovano in conflitto, privilegiando il buon andamento della giustizia a scapito del diritto di cronaca, che subisce semplicemente un limite ristretto a determinati casi. Ma si tratta di orientamento oggi prevalente e che domani potrà non essere più condiviso: già si percepiscono le prime avvisaglie della estensione anche all'art. 684 c.p. di una opinione nata per la diffamazione, secondo la quale il diritto di cronaca può essere esercitato anche quando viene a ledere un altro diritto (nella specie: l'altrui reputazione), qualora vengano rispettati i limiti della verità del fatto narrato, $l^{\prime}$ interesse attuale e pubblico alla divulgazione del fatto, nonchè la continenza della forma espressiva. Ora, facile comprendere che l'estensione di questo orientamento all'art. 684 c.p. ne vanificherebbe quasi del tutto la portata.

Se la divulgazione delle notizie avviene ad opera di magistrati, cancellieri, periti, polizia giudiziaria e così via, la fattispecie applicabile è la rivelazione ed utilizzazione di segreti d'ufficio di cui all'art. 326 c.p.: "Il pubblico ufficiale o la persona incaricata di un pubblico servizio, che, violando i doveri inerenti alle funzioni o al servizio, o comunque 
abusando della sua qualità, rivela notizie d'ufficio, le quali debbano rimanere segrete, o ne agevola in qualsiasi modo la conoscenza, è punito con la reclusione da sei mesi a tre anni. Se l'agevolazione è soltanto colposa, si applica la reclusione fino a un anno.

Il pubblico ufficiale o la persona incaricata di un pubblico servizio, che, per procurare a sé o ad altri un indebito profitto patrimoniale, si avvale illegittimamente di notizie $\mathrm{d}^{\prime}$ ufficio, le quali debbano rimanere segrete, è punito con la reclusione da due a cinque anni. Se il fatto è commesso al fine di procurare a sé o ad altri un ingiusto profitto non patrimoniale o di cagionare ad altri un danno ingiusto, si applica la pena della reclusione fino a due anni". Se alla rivelazione segue la pubblicazione, ci si pone il problema del concorso di reati o del concorso apparente di norme tra rivelazione e pubblicazione, qualora vi sia un' unica condotta. Per coloro che operano all'interno del processo, la rivelazione di atti che devono rimanere segreti comporta una sanzione pesante. Ma l'applicazione di questa norma è scarsa o, per lo meno, non corrisponde alla frequenza con cui il segreto è violato.

L'art. 326 c.p. non opera per i difensori, le parti private, i consulenti, i testimoni e le persone informate sui fatti, alle quali si applica l'art. 379 bis c.p.: "Rivelazione di segreti inerenti a un procedimento penale". La norma recita:"Salvo che il fatto costituisca più grave reato, chiunque rivela indebitamente notizie segrete concernenti un procedimento penale, da lui apprese per avere partecipato o assistito ad un atto del procedimento stesso, è punito con la reclusione fino a un anno. La stessa pena si applica alla persona che, dopo avere rilasciato dichiarazioni nel corso delle indagini preliminari, non osserva il divieto imposto dal pubblico ministero ai sensi dell'articolo 391- quinquies del codice di procedura penale". Questa non è l'unica norma in questo 
senso: nel codice di procedura penale ci sono vari divieti, che seguono le diverse fasi del procedimento e poi del processo.

Esistono inoltre norme di deontologia: il testo unico dei doveri del giornalista del 2016 impone il rispetto della presunzione di innocenza e impone che sia sempre reso chiaro ciò che viene documentato e ciò che invece ne costituisce il commento. Solo questo, se fosse osservato, basterebbe ad evitare i problemi di commistione tra il processo mediatico e quello giuridico.

C'è poi una Raccomandazione del Consiglio d'Europa del 2003 che sancisce un dovere di informazione a mezzi di comunicazione da parte dell'autorità giudiziaria e di polizia, in rapporto a procedimenti penali di pubblico interesse, purché ciò non comprometta il segreto investigativo e le indagini di polizia. Insomma, il diritto di dare informazione $c^{\prime}$ è, ma non si può compromettere il segreto investigativo. Il principio 10 di questa raccomandazione determina che "in rapporto a processi penali, soprattutto qualora vi siano coinvolti giurati o giudici onorari, le autorità giudiziarie e di polizia dovrebbero evitare di fornire pubblicamente informazioni che comportino il rischio di pregiudicare in misura sostanziale la correttezza del procedimento". Concetto che viene considerato così importante da far dire al principio 11 che "qualora la persona accusata di un reato sia in grado di dimostrare che le informazioni fornite comportano una probabilità elevata di ledere il suo diritto ad un giusto processo, o hanno già dato luogo a tale lesione, la persona in oggetto dovrebbe disporre di un rimedio giuridico efficace". Poiché, infatti, soprattutto i giudici non togati possono essere influenzati dalle notizie che ricevono fuori dal processo e dagli atti processuali, è necessario che si preveda un rimedio per la parte lesa. Una specie di moderno guidrigildo. 
Se guardiamo, poi, alle sentenze della Corte europea dei diritti dell'uomo ci rendiamo conto che realizzano proprio il bilanciamento necessario tra interessi contrapposti perché aprono all'idea di processo mediatico ma impongono limiti. In alcune pronunce si sostiene, infatti, che se il compito dei tribunali è quello di comporre le controversie, nulla vieta che le stesse controversie possano dar luogo a dibattiti in altre sedi. E non basta. E' diritto del pubblico essere informato ed è compito dei giornalisti divulgare ciò di cui i tribunali si occupano. Ma questa informazione va svolta con la discrezione imposta dalla presunzione di innocenza, stabilita anche dall' art. 6 della Convenzione europea dei diritti dell'uomo. Sembrerebbe, ancora una volta, un richiamo all'equilibrio.

La Corte di Strasburgo ha sanzionato i comportamenti scorretti di quei protagonisti del processo rituale che non ne rispettano i principi. Per esempio, ha sanzionato l'accusa quando ha rappresentato l'indagato o imputato come sicuro colpevole del crimine di cui lo si incolpa ${ }^{27}$. Oppure ha censurato la difesa ${ }^{28}$ quando gli avvocati si sono abbandonati a censure rivolte a singoli magistrati con gravità e toni incompatibili col tributo che gli avvocati sono chiamati ad apportare alla fiducia del popolo nella giustizia.

Insomma, la conclusione che possiamo trarre è che non ci troviamo in una terra senza legge ma in un ambito regolamentato in maniera, forse non del tutto sufficiente, ma comunque chiara.

\section{La realtà.}

Malgrado l'esistenza delle norme atte ad evitare i rischi del processo mediatico, restano pericolose le modalità

27 Allenet de Ribemont vs. Francia, Application no. 15175/89, 10/2/1995.

28 Schopfer vs. Svizzera, (56/1997/840/1046) 20/5/1998. 
fortemente suggestive con cui spesso l'informazione interviene sui temi legati alla giustizia, tanto da avere spinto l'Osservatorio delle Camere Penali a pubblicare un Libro bianco sull'informazione giudiziaria in Italia ${ }^{29}$. Pur non dimenticando che le Camere Penali sono comunque un organo corporativo che riunisce gli avvocati penalisti, quantitativamente più inclini alla difesa degli indagati ed imputati piuttosto che a quella della parte civile, vale la pena dar conto dei principali problemi che sono stati rilevati durante questa ricerca. In primo luogo è stata evidenziata nei racconti presentati dai media relativi a fatti penalmente rilevanti una sorta di acquiescenza pregiudiziale alle tesi accusatorie, così come un distacco dal potere giudiziario, considerato spesso come un' estensione di quel male assoluto che è oggi la politica. Entrambi questi rilievi colgono, almeno in parte, nel segno. Anche semplicemente da un punto di vista quantitativo, è molto più facile trovare una cronaca mediatica di un determinato fatto criminoso- non solo di sangue- che sposi la tesi accusatoria piuttosto che fornisca elementi di difesa. La ricerca di un colpevole a tutti i costi soddisfa l'esigenza di quella giustizia sommaria priva di garanzie che appaga il sentimento popolare. Fino a quando non si rimanga coinvolti in qualche vicenda giudiziaria: in quel momento ci si lamenterà della mancanza di garanzie e della apparente certezza con cui i media attribuiscono colpe e responsabilità. Ma di certo le tesi accusatorie meglio soddisfano macabre curiosità e pruderie del pubblico.

Altrettanto vero, poi, è che in un periodo storico in cui i cittadini si distanziano dalla politica e prendono di mira i privilegi, il potere giudiziario, che incarna comunque il senso di un potere amministrato in nome dello Stato (mentre è esercitato, in realtà, in nome del popolo) è visto con diffidenza.

29 Libro bianco sull'informazione giudiziaria in Italia del 21/11/2016 
Si preferisce una giustizia magari meno raffinata, ma che si basa sul senso comune di tutti i cittadini, anche -meglio: soprattutto- se nulla sanno dei meccanismi giuridici che presiedono al diritto penale. Meccanismi, come abbiamo visto, non legati ad inutili forme ma che sono il portato di lunghi anni in cui le garanzie dell'imputato si sono fatte breccia nel sistema inquisitorio.

Qualunque consorzio umano vuole conoscere e giudicare, per punirli, quei comportamenti che ritiene incompatibili con la sopravvivenza dello stesso. Ed è proprio per questo che, nei secoli, sono state individuate procedure che consentano di arrivare alla verità. Il processo dovrebbe proprio essere quel percorso con cui un soggetto terzo passa dalla res judicanda alla res judicata e che consente alla società di accettare quest' ultima come verità. Ma negli ultimi tempi è proprio la res judicata ad essere messa in discussione, se non si adegua a quel sentimento popolare che mira ad accertare, con le sue forme e metodi, la stessa verità. Il problema è che i responsi che vengono da un procedimento condiviso consentono di assorbire i rischi di radicalizzazione del dissenso ed oggi è proprio questo dissenso che ci si trova a dover fronteggiare, visto che il processo celebrato nelle aule di giustizia non si pone più come quella procedura condivisa ed accettata. Ciò che preoccupa è che l'usuale procedimento democratico per arginare il dissenso sarebbe che la collettività, attraverso i suoi rappresentanti politici, cambiasse le regole di accertamento per renderle condivise. Questo, ovviamente, postula che la collettività possa conoscere come viene pronunciato il diritto e questo dipende anche dai mass media, che però dovrebbero avere funzione di ausilio e non di sostituzione. Il che, evidentemente, non è.

Facendo un passo indietro, due sono i momenti di intersezione tra processo penale ed informazione. Il primo è 
l'informazione sul processo; il secondo è il processo celebrato dai mezzi di informazione, spesso riproducendo le liturgie del processo in un foro alternativo. Lo abbiamo già detto ma vale la pena ripeterlo. La cronaca del processo è essenziale per rispettare il principio secondo cui la giustizia è amministrata in nome del popolo. Fare cronaca non significa solo dare la notizia senza accenni critici o spunti di riflessione diversi da quelli sollecitati nel processo. Tuttavia si deve fuggire da un processo parallelo. Da evitare, infatti, è proprio che l'accertamento della verità si snodi su più binari paralleli. Binari che seguono regole molto diverse ${ }^{30}$ : basti pensare ad alcuni aspetti per comprendere le differenze di approdi cui i due tipi di accertamento possono condurre. Mentre il processo è scandito da un rigoroso iter all'interno di un luogo deputato e finisce col giudicato, il processo mediatico non segue alcun ordine, può celebrarsi dovunque ed è senza tempo. Il processo ordinario è celebrato da un organo professionale, seleziona i dati su cui può fondare la decisione e li valuta con criteri che sono il frutto di regole di esperienza; il processo mediatico è officiato da qualcuno che raccoglie in modo bulimico le informazioni e non le seleziona. Il modello del codice di procedura penale vigente è tendenzialmente accusatorio, basato sulla parità tra accusa e difesa, mentre il processo mediatico è totalmente inquisitorio e valgono solo intuizioni, buon senso ed emotività. Alla logica del probabile si sostituisce la logica dell'apparenza: ciò che sembra, facilmente sarà. Ciò che conta è il convincimento collettivo.

Il vero problema è che l'utente non sempre riesce a tenere distinti i due ambiti ed, anzi, quando accade che vengano messi a confronto, la sentenza che deriva dall'applicazione delle regole del processo giudiziario è quella considerata meno vera. Quasi come se le regole che al processo presiedono,

30 Si veda Glauco Giostra, Processo penale e mass media, in Criminalia, 2007 
invece di costituire garanzia di giustizia, frapponessero un velo fumoso tra i fatti e le conseguenze punitive. E' come se ci fosse una sorta di diffidenza nei confronti della giustizia tradizionale, che appare come troppo macchinosa. Continua a saltare agli occhi il paragone con la attuale sfiducia dei cittadini nella politica: è come se ogni potere fosse rinnegato per il fatto stesso di essere un potere. Se è vero che la giustizia deve essere esercitata in nome del popolo, va rilevato che oggi è raro -se non in alcuni casi- che il cittadino vada in aula per assistere al processo; più facilmente è il processo che va da lui, entra nella sua casa. Se da cittadini scegliamo che cosa seguire, da spettatori non possiamo che subire una selezione ideologica di notizie. Però resta il fatto positivo, per nulla trascurabile, che l'esercizio della giustizia è ora maggiormente aperto alla conoscenza dei più. Il tribunale dell'opinione pubblica risponde ad una regola elementare di psicologia comportamentale: la prospettiva di una verifica esterna del proprio operato dissuade da abusi e devianze. Ma anche questo aspetto positivo ha il suo contraltare: chi va ad assistere ad un processo nelle aule di giustizia difficilmente avrà modo di incidere su questo, mentre i mass media hanno la capacità di condizionare l'oggetto che rappresentano. Le distorsioni non riguardano il singolo processo ma l'intero sistema.

Altro aspetto significativo è che si crea una distorsione dovuta al fatto che ci vengono rappresentati dai mass media solo quei processi nei quali qualcuno ha avuto l'interesse di una fuga di notizie. Fuga che è stata poi valutata dal giornalista che ha deciso oppure no la divulgazione. Un doppio filtro di selezione che già $a b$ origine orienta in un senso $\mathrm{o}$ nell'altro e, in entrambi i sensi, ci sarà una determinata incidenza sul processo. 
Certo, innumerevoli sono anche i casi di iniziative giudiziarie portate avanti proprio grazie ad inchieste giornalistiche che hanno evitato insabbiamenti. Infatti, una giustizia segreta sarebbe impensabile in un ordinamento democratico.

Insomma: la situazione è complessa e ci sono commistioni tra elementi del processo mediatico che svolgono una funzione pubblica ed elementi -la maggior parte- che imporrebbero un uso più attento del diritto di cronaca, che in realtà arriva a sconfinare nelle mere supposizioni. Siamo in una zona grigia, dove non ci sono aspetti solo negativi o solo positivi ma tutto deve essere giocato sull'equilibrio. Oppure -come vedremo nel prossimo paragrafo- sulla accettazione delle differenze.

\section{Le verità.}

Abbiamo appurato, attraverso la breve disamina svolta, che il processo mediatico è un mostro a tratti necessario ed ormai inevitabile. Le soluzioni prospettabili per porre fine alle distorsioni che il processo celebrato dai mezzi di informazione porta con sè sono molte. Bisognerebbe poter contare sulla professionalità di tutti i soggetti coinvolti: quelli del processo ordinario e quelli del processo mediatico. Un utilizzo attento delle norme sulla violazione del segreto nelle varie fasi processuali aiuterebbe la fuga di notizie. Un richiamo deontologico alle figure del processo ordinario che frequentano i programmi in cui si svolgono i processi mediatici di certo aiuterebbe un esercizio della giustizia svolto nelle sedi opportune. Ma anche questa indicazione non è univoca e potrebbe essere letta in senso opposto: la presenza di difensori o altri protagonisti del processo rituale in quello mediatico potrebbe essere vista come garanzia che quest'ultimo si svolga senza stravolgimenti della realtà. 
Nessuna situazione univoca, quindi. Appena indicazioni di massima, oltretutto di scarsa utilità.

Il richiamo alla professionalità dei giornalisti potrebbe essere un dato importante quando si abbia a che fare con una informazione adeguata, di sicuro non quando il livello dell'informazione prodotta sia scadente e ci si basi sul clamore per ottenere la pubblicità che serve per mantenere viva la testata. Com'è tipico di molti periodici on line, spesso molto seguiti a livello locale. Educare il cittadino a differenziare il tipo e la qualità dell'informazione senza dubbio porterebbe ad una maggior consapevolezza critica, ma tutto quello che riguarda l'educazione necessita di tempo e risorse. Utilissima sarebbe un' educazione al controllo della fonte giornalistica ed al confronto dei mezzi di informazione per un continuo miglioramento del prodotto informativo. Antidoto alle distorsioni è senz'altro una informazione libera e plurale, con giornalisti capaci e professionali.

Insomma, tutti quelli elencati -e potrebbero essercene molti altri- sono rimedi che possono contribuire a risolvere il problema, ma nessuno di questi mi pare risolutivo nè immediatamente operativo. Non ci resta che rassegnarci ad un'evoluzione -forse meglio: involuzione- della giustizia che si trova a fare i conti anche con gli umori che vengono dall'esterno. Soluzione apparentemente arrendevole ma che potrebbe avere un fondamento teorico-filosofico. Forse si rende necessario un mutamento delle categorie concettuali, il che si porrebbe anche in linea con gli approdi più recenti della teoria della scienza, secondo cui la conoscenza procede per rotture e cambiamenti, non attraverso un percorso lineare ${ }^{31}$.

Da tempo siamo abituati al fatto che la verità storica e quella processuale non coincidono. Ciò che è realmente

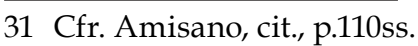


successo non sempre si sovrappone alla ricostruzione che dell' accaduto vien fatta nei tribunali, attraverso la raccolta delle prove rituali. D'altronde, storico e giurista hanno metodi diversi, anche se per molti aspetti simili. Anche la storia si fa attraverso le testimonianze, perché attingere alla realtà in presa diretta è impossibile, così come è impossibile farlo nel processo penale. Anche la storia spiega gli accadimenti "in funzione di" e non semplicemente attraverso l'accaduto. Storici e giudici usano le prove perché il mestiere di entrambi è provare, in base a determinate regole, che "Tizio ha fatto quello". In entrambi i casi il risultato della prova resta nell'ordine della probabilità, ma con livelli diversi: sebbene non possa richiedersi la certezza, nel processo si richiede che il fatto sia provato oltre il ragionevole dubbio. Senz'altro diverso, invece, l'obbligo di tenere in conto le garanzie dei diritti dell'imputato, che grava sul giurista e non sullo storico ${ }^{32}$.

Insomma: siamo ormai abituati allo scarto tra la verità storica e quella processuale, benché quest'ultima debba sempre avere come fine la ricostruzione di ciò che realmente è accaduto, cioè deve avere come orizzonte quello della verità storica.

E qui si inserisce un' osservazione che potrebbe apparire eretica. Il concetto di verità, tradizionalmente visto come monolitico e quasi trascendente, non è unico. Non c'è una sola verità, ce ne sono molte. Sembrerebbe quasi un ossimoro: storicamente la verità, per sua natura, non può che essere unica. Ma se si guarda alle entità delle quali può predicarsi l'aggettivo "vero", ci si rende conto che questo può dirsi di entità eterogenee: per esempio, di affermazioni e di cose. Il verus de dicto ed il verus de re ${ }^{33}$. La verità de dicto è quella apo-

32 Cfr. Ginzburg, Il giudice e lo storico, Einaudi, 1991, 13ss.

33 Conte, Tres vidit. Verità apofantica, verità eidologica, verità idiologica, in Cavalla, 
fantica, collegata, cioè, al contenuto cognitivo ed al rapporto con la realtà. La filosofia analitica privilegia la verità de dicto perchè si riferisce ad enunciati, ma esiste anche quella de re che si pratica su entità semiotiche come norme, domande, nomi. E la verità de re può essere eidologica ${ }^{34}$, quando la sua corrispondenza all'entità cui si riferisce è generica, oppure idiologica, quando questa corrispondenza non avviene con un eidos ma con un' entità individuale. Senza entrare troppo nel dettaglio, quel che conta è che non esiste un'unica dimensione di verità.

Calando queste osservazioni nel discorso che stiamo portando avanti, ciò che è accaduto è incontestabile ma non lo è la sua ricostruzione storica. Quando, poi, la ricostruzione si basa su elementi tipici e per ciò stesso selezionati, come le prove, le difficoltà aumentano. Non si dimentichi, poi, il gioco delle parti, per le quali il processo è un laboratorio il cui punto d'avvio è la topica, intesa come "l'arte di trovare le premesse per l'esercizio della dialettica le cui conclusioni, ove sia il caso, vengono diffuse pervasivamente attraverso la retorica" ${ }^{35}$. Certo non si tratta di retorica strategica bensì rigorosa, proprio a causa delle regole che presiedono al processo penale. Ma non si tratta neppure di verità scientifica, perché non muove da premesse indiscusse. Ad ogni modo, la dialettica processuale mira all' $\varepsilon^{\prime} v \delta o \xi a$, cioè alla verità degli argomenti utilizzati. Al contrario,la retorica utilizzata nel processo mediatico subordina la verità degli argomenti utilizzati alla loro capacità persuasiva: mira, infatti, al пعı $\theta \dot{\omega}$,

Retorica, processo, verità, Milano, 2007, pag.298ss.; id. Adelaster. Il nome del vero, Led, Milano, 2016

34 Conte, Adelaster, cit., 15ss.

35 Cavalla, Retorica processo verità. Principi di filosofia forense, FrancoAngeli, 2007, $57 \mathrm{ss}$ 
alla persuasione. Ecco che il metodo non può non avere conseguenze sul tipo di verità che si ottiene.

Ma anche guardando al tipo di giustizia che si vuole ottenere, è inevitabile lo scarto tra la giustizia normativa, che è quella delle leggi e la giustizia reale, amministrata nei tribunali. A questo potremmo aggiungere anche lo scarto con la giustizia rappresentata, che è quella raccontata dai mezzi di informazione.

Mezzi, fini, modalità diverse. Verità diverse.

Ebbene, forse allora dovremmo abituarci alla compresenza di tre verità: quella storica, quella processuale ed infine quella mediatica. La verità storica intesa come ciò che è realmente accaduto si affianca a quella processuale, ricostruita attraverso i mezzi processuali e che determina oppure no l'applicazione di una sanzione penale. Ed a queste si aggiunge la verità mediatica, ottenuta con i mezzi mediatici ed il cui impatto resta emotivo e sociale. Come dicevamo: mezzi, fini, modalità diverse. Verità diverse.

La consapevolezza di queste tre verità, che sono e restano distinte, insieme ad un'alta professionalità di tutti i protagonisti del processo mediatico potrebbe forse contribuire ad evitare gli effetti distorsivi del processo svolto fuori dalle aule giudiziarie. 


\section{Referencias}

Arendt, La banalità del male. Eichmann a Gerusalemme, Feltrinelli, 2013

Bertolino, La televisione del crimine, in Vita \& Pensiero, 2005

Cavalla, Retorica processo verità. Principi di filosofia forense, FrancoAngeli, 2007

Cheli, La realtà mediata. L'influenza dei mass media tra persuasione e costruzione sociale della realtà, Franco Angeli, Milano, 1992 Conte, Tres vidit. Verità apofantica, verità eidologica, verità idiologica, in Cavalla, Retorica, processo, verità, Milano, 2007, pag.298ss.

Conte, Adelaster. Il nome del vero, Led, Milano, 2016

Garland, La cultura del controllo. Crimine e ordine sociale nel mondo contemporaneo, Il Saggiatore, 2007

Giostra, Processo penale e mass media, in Criminalia, 2007

Luhmann, La realtà dei mass media, Franco Angeli, Milano, 2000

Moccia, Politica criminale e riforma del sistema penale, Jovene, Napoli, 1984

Moccia, La perenne emergenza. Tendenze autoritarie nel sistema penale, Esi, Napoli, 2000

Paliero, La maschera e il volto, in Riv. it. dir e proc pen., 2006, n. 2,

Roxin, Politica criminale e sistema del diritto penale, Esi, Napoli, 2001

Silverstone, Perchè studiare i media?, Il Mulino, Bologna, 2002, p. 7ss. 
Sorrentino, Storia del processo penale. Dall'ordalia all'inquisizione, Rubbettino, 1999

Wolf, Teorie delle comunicazioni di massa, Strumenti Bompiani, 2001

Recebido em 13/09/2018.

Aprovado em 13/09/2018.

Maristella Amisano

E-mail:maristami@libero.it 